\title{
An Efficient Approach to Intensity Inhomogeneity Compensation Using $c$-Means Clustering Models ${ }^{\star}$
}

\author{
László Szilágyi, David Iclănzan, Lehel Crăciun, and Sándor Miklós Szilágyi \\ Sapientia - Hungarian Science University of Transylvania, \\ Faculty of Technical and Human Science, Tîrgu-Mureş, Romania \\ lalo@ms.sapientia.ro
}

\begin{abstract}
Intensity inhomogeneity or intensity non-uniformity (INU) is an undesired phenomenon that represents the main obstacle for magnetic resonance (MR) image segmentation and registration methods. Various techniques have been proposed to eliminate or compensate the INU, most of which are embedded into clustering algorithms, and they generally have difficulties when INU reaches high amplitudes. This study reformulates the design of $c$-means clustering based INU compensation techniques by identifying and separating those globally working computationally costly operations that can be applied to gray intensity levels instead of individual pixels. The theoretical assumptions are demonstrated using the fuzzy $c$-means algorithm, but the proposed modification is compatible with a various range of $c$-means clustering based techniques. Experiments using synthetic phantoms and real MR images indicate that the proposed approach produces practically the same segmentation accuracy as the conventional formulation, but 20-30 times faster.
\end{abstract}

Keywords: image segmentation, magnetic resonance imaging, intensity inhomogeneity, c-means clustering, histogram.

\section{Introduction}

Magnetic resonance imaging (MRI) is popular due to its high resolution and good contrast. However, the automatic segmentation of such images is not trivial because of the noise that may be present. Intensity inhomogeneity or intensity non-uniformity (INU) represents an undesired phenomenon in MRI, manifested as a slowly varying bias field with possibly high magnitude, making pixels belonging to the same tissue be observed with different intensities. INU is the main obstacle for intensity based segmentation methods: several efficient and accurate removal techniques exist for high frequency noise [13, but the segmentation in the presence of inhomogeneities represents a significant computational load [16].

Inhomogeneities in magnetic resonance (MR) images are usually categorized by their origin. Device related INU artifacts can be efficiently compensated via

\footnotetext{
* This research was funded by CNCSIS UEFISCDI, project no. PD_667, under contract no. 28/05.08.2010.
} 
calibration methods based on prior information obtained by using a uniform phantom. Alternately, INU artifacts related to the shape, position, structure and orientation of the patient, are much more difficult to handle [16]. Several retrospective INU compensation approaches have been reported, which include homomorphic filtering [5], polynomial or B-spline surface fitting [14, segmentation based techniques via maximum likelihood estimation [9], Markov random fields [17, fuzzy $c$-means clustering [1810, or nonparametric estimation 3]. Further INU compensation procedures based on histogram involve high-frequency maximization [11, information maximization [15], or histogram matching [12]. The most complete review of INU compensation techniques can be found in [16].

Probably the most widely used compensation tool is the fuzzy $c$-means (FCM) algorithm 2, having several adaptations for INU estimation and being combined with a series of further techniques. Pham and Prince introduced a modified objective function producing bias field estimation and containing extra terms that force INU vary smoothly 8 . Liew and Hong created a log bias field estimation technique that models the INU with smoothing B-spline surfaces [6. Ahmed et al. established a regularization operator that allowed the labeling of a pixel to be influenced by its immediate neighbors [1]. This approach reduced some of the complexity of its ancestors, but the zero gradient condition that was used for bias field estimation leads to several misclassifications [10].

The compensation of INU artifacts is a computationally costly problem, which demands highly efficient design and implementation. This paper demonstrates that the INU compensation on a single-channel intensity image via $c$-means clustering can be performed much more efficiently than it was reported in previous formulations. The operations performed during the iterations of the alternating optimization (AO) scheme are separated into globally working ones and locally applied ones, and their execution is optimized according to their necessities: global criteria are applied to gray intensities instead of individual pixels, which makes a drastic reduction of the computational load. Using this novel formulation, and applying it to improved clustering models (e.g. 7[13]) combined with multi-stage INU compensation, can make $c$-means clustering more attractive on the combined scales of accuracy and efficiency. Improving the accuracy is not in the scope of this paper. Our main goal is to reduce the execution time without damaging the accuracy.

\section{Background Works}

The conventional FCM algorithm optimally partitions a set of object data into a previously set number of $c$ clusters based on the iterative minimization of a quadratic objective function. When applied to segment gray-scale images, FCM clusters the intensity value of pixels $x_{k}, k=1 \ldots n$. The objective function

$$
J_{\mathrm{FCM}}=\sum_{i=1}^{c} \sum_{k=1}^{n} u_{i k}^{m}\left(x_{k}-v_{i}\right)^{2}
$$


is optimized under the so-called probability constraint $\sum_{i=1}^{c} u_{i k}=1, k=1 \ldots n$, where $u_{i k} \in[0,1]$ is the fuzzy membership function indicating the degree to which pixel $k$ is assigned to cluster $i, v_{i}$ represents the centroid or prototype of the $i$-th cluster, and $m>1$ is the fuzzy exponent. The minimization of the cost function is reached by alternately applying the optimization of $J_{\mathrm{FCM}}$ over $\left\{u_{i k}\right\}$, $i=1 \ldots c, k=1 \ldots n$ with $v_{i}$ fixed, and the optimization of $J_{\mathrm{FCM}}$ over $\left\{v_{i}\right\}$, $i=1 \ldots c$, with $u_{i k}$ fixed [2].

In real data processing, the observed data $\left\{y_{k}\right\}$ differs from the actual one $\left\{x_{k}\right\}$. In this paper we only assume to handle the INU artifacts, by compensating during segmentation. Literature recommends three different data variation models for intensity inhomogeneity. If we consider the INU as a bias field, for any pixel $k$, we will have $y_{k}=x_{k}+b_{k}$, where $b_{k}$ represents the bias value at pixel $k$ [1810. In case of gain field modeling [13, there will be a gain value $g_{k}$ for each pixel $k$, such that $y_{k}=g_{k} x_{k}$. Finally, the so-called log bias approach in fact is a gain field estimation reduced to bias computation using the logarithmic formula $\log y_{k}=\log g_{k}+\log x_{k}$ [6]. Regardless of the used compensation model, the variation of the intensity between neighbor pixels has to be slow. The zero gradient conditions derived from FCM's objective function does not fulfil this demand. Consequently, a smoothing operation is necessary to assure this slow variation of the estimated bias or gain field.

In the INU compensation problem, the conventional FCM based approach optimizes the objective function:

$$
J_{\mathrm{FCM}-\mathrm{b}}=\sum_{i=1}^{c} \sum_{k=1}^{n} u_{i k}^{m}\left(y_{k}-b_{k}-v_{i}\right)^{2} .
$$

Zero gradient conditions and Lagrange multipliers lead to the following optimization formulas. The fuzzy partition is obtained as:

$$
u_{i k}^{\star}=\frac{\left(y_{k}-b_{k}-v_{i}\right)^{-2 /(m-1)}}{\sum_{j=1}^{c}\left(y_{k}-b_{k}-v_{j}\right)^{-2 /(m-1)}} \quad \forall k=1 \ldots n, \quad \forall i=1 \ldots c .
$$

Cluster prototypes are updated as:

$$
v_{i}^{\star}=\frac{\sum_{k=1}^{n} u_{i k}^{m}\left(y_{k}-b_{k}\right)}{\sum_{k=1}^{n} u_{i k}^{m}} \quad \forall i=1 \ldots c .
$$

The bias field for the pixel $x_{k}$ is estimated as:

$$
b_{k}^{\star}=y_{k}-\frac{\sum_{i=1}^{c} u_{i k}^{m} v_{i}}{\sum_{i=1}^{c} u_{i k}^{m}} \quad \forall k=1 \ldots n .
$$

\section{Methodology}

When a clustering algorithm is required to perform quickly on a large set of input data, the aggregation of similar input values is an easily implementable 
choice. It is well known, that the FCM algorithm in image processing belongs to the segmentation methods that work with global information. This means that pixels will be assigned to clusters based on their own intensity (color), without regard to their position in the image. Consequently, pixels with same intensity will belong to the same clusters with the same membership degrees. Based on this assumption, it is obvious that the FCM-based segmentation of single-channel intensity image can be performed based on the histogram, by clustering the colors instead of individual pixels [13].

Table 1. The proposed accelerated algorithm

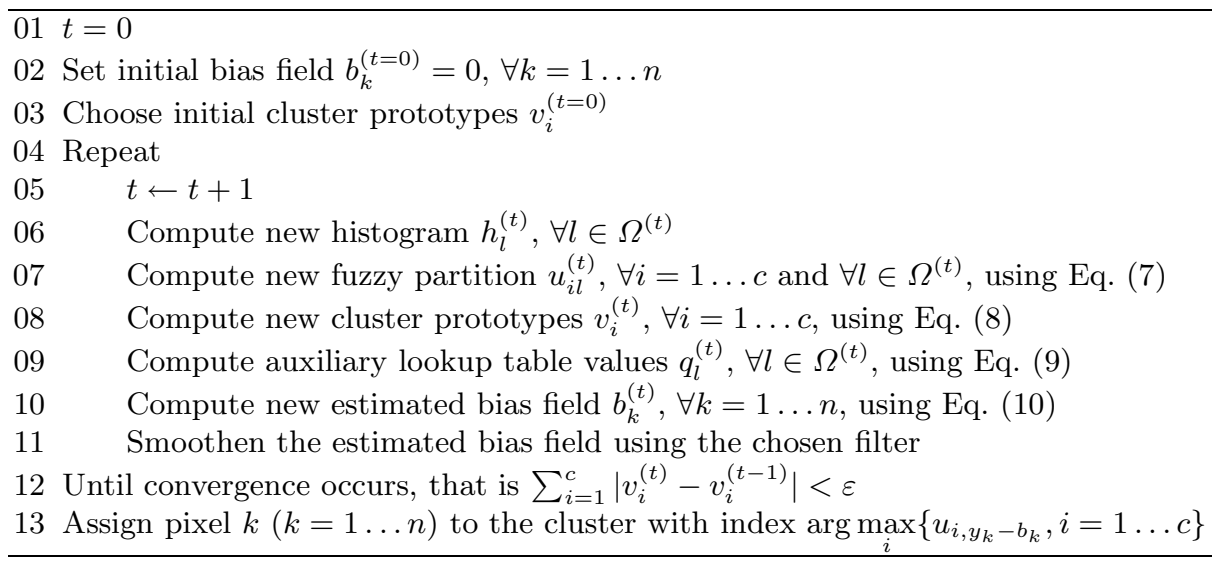

When INU artifacts are present, local conditions must be involved into the compensation process, in order to assure the smooth variation of the estimated inhomogeneity. Consequently, pixels of similar or same observed intensity cannot be collected and handled together. In the followings, we will demonstrate that most operations of the INU compensation algorithm can be executed using global information, which will lead to a drastic reduction of the computational load.

Let us consider the cost function of the bias estimation approach, given in Eq. (2). The input image contains pixels in order of $10^{4}-10^{5}$, and intensity levels in order of $10^{2}-10^{3}$. In every iteration of the $\mathrm{AO}$ algorithm, we need to aggregate those pixels, which bear the same intensity after having the current estimated bias subtracted. That is why, we investigate the distribution of the composite variable $y_{k}-b_{k}$, which varies from iteration to iteration. Let us denote by $h_{l}^{(t)}$ the number of pixels for which the compensated intensity in iteration $t$ satisfies $y_{k}-b_{k}=l$. Obviously, if we denote by $\Omega^{(t)}$ the range of possible values of $y_{k}-b_{k}$, we will have $\sum_{l \in \Omega^{(t)}} h_{l}^{(t)}=n$. As the matter of fact, $h_{l}^{(t)}$ with $l \in \Omega^{(t)}$ represents the intensity histogram of the compensated image in iteration $t$.

Using the above notations, we can aggregate equal values of $y_{k}-b_{k}$ in the cost function, which in iteration $t$ will become:

$$
J_{\mathrm{FCM}-\mathrm{qb}}=\sum_{i=1}^{c} \sum_{l \in \Omega^{(t)}} h_{l}^{(t)} u_{i l}^{m}\left(l-v_{i}\right)^{2} .
$$


Zero gradient conditions and Lagrange multipliers lead to the following optimization formulas. Fuzzy memberships are established as

$$
u_{i l}^{\star}=\frac{\left(l-v_{i}\right)^{-2 /(m-1)}}{\sum_{j=1}^{c}\left(l-v_{j}\right)^{-2 /(m-1)}} \quad \forall l \in \Omega^{(t)} \quad \forall i=1 \ldots c .
$$

One evaluation of the above formula computes the fuzzy labels of $h_{l}^{(t)}$ pixels at the same time. Cluster prototypes are updated as:

$$
v_{i}^{\star}=\frac{\sum_{l \in \Omega^{(t)}} h_{l}^{(t)} u_{i l}^{m} l}{\sum_{l \in \Omega^{(t)}} h_{l}^{(t)} u_{i l}^{m}} \quad \forall i=1 \ldots c .
$$

This formula is evaluated $c$ times in every iteration, like in case of conventional FCM-b, but here both the denominator and divisor of the fraction sum up much fewer terms. Obviously the estimated bias field has to treat each pixel separately. But even here we can simplify the computations by introducing some auxiliary variables and organizing them into a lookup table. In this order, let

$$
q_{l}=\frac{\sum_{i=1}^{c} u_{i l}^{m} v_{i}}{\sum_{i=1}^{c} u_{i l}^{m}} \quad \forall l \in \Omega^{(t)},
$$

and subsequently, for any pixel with index $k=1 \ldots n$, we get the estimated bias:

$$
b_{k}^{\star}=y_{k}-q_{l_{k}} \quad \text { with } \quad l_{k}=y_{k}-b_{k}^{(t-1)} .
$$

The proposed accelerated algorithm is summarized in Table 1

\section{Results and Discussions}

The theoretical time complexity of the conventional and accelerated approach is compared in Table 2, where $\omega$ stands for the cardinality of the set $\Omega$ or the number of different intensity values in the current compensated image. Considering the fact that the number of present gray intensities $(\omega)$ is less than the number of pixels $(n)$ in the image by minimum two orders of magnitude, we can state that the time consuming first three steps of the conventional algorithm are replaced with much quicker solutions in the proposed algorithm.

Both the conventional and proposed approaches were tested on 24 artificial phantoms and 40 real MR images. Artificial phantoms were created by adding slowly varying INU noise to single-channel intensity images that contained two easily separable regions of constant intensity. Real MR images were taken from the Internet Brain Segmentation Repository [4]. All benchmark results were obtained on a PC with Athlon64 processor running at $2 \mathrm{GHz}$ frequency.

Figure 1(a) summarizes the benchmark results of both algorithms that were executed on artificial phantoms of various sizes. In case of two classes, the proposed algorithm accelerates the execution about 15-25 times. It is also visible that the speed-up ratio slightly rises if we increase the size of the input phantom 
Table 2. Computational complexity of algorithmic steps

\begin{tabular}{l|cc}
\hline Algorithmic step & Conventional (FCM-b) & Accelerated (FCM-qb) \\
\hline Partition updating & $\mathcal{O}\left(n c^{2}\right)$ & $\mathcal{O}\left(\omega c^{2}\right)$ \\
Cluster prototype updating & $\mathcal{O}(n c)$ & $\mathcal{O}(\omega c)$ \\
Bias estimation & $\mathcal{O}(n c)$ & $\mathcal{O}(n+\omega c)$ \\
Bias smoothing & $\mathcal{O}(n)$ & $\mathcal{O}(n)$ \\
Histogram updating & - & $\mathcal{O}(n)$ \\
\hline
\end{tabular}

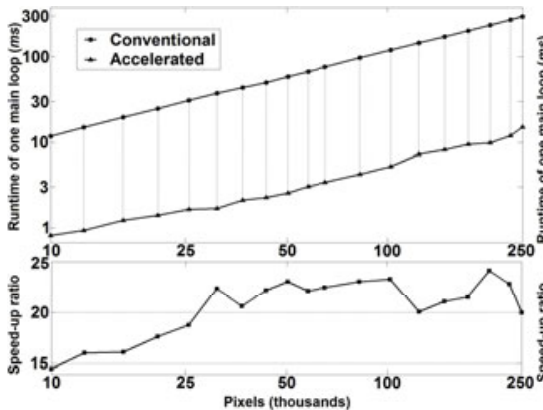

(a)

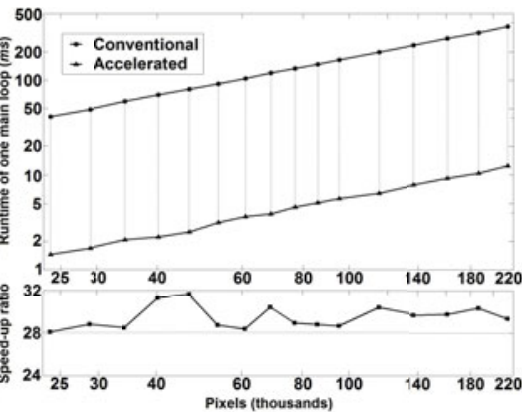

(b)

Fig. 1. Runtime of one main loop, using the conventional and accelerated approach (up), and the resulting speed-up ratio (down), all represented against the number of pixels in the image: (a) phantom images, segmented into $c=2$ classes; (b) real MR images, segmented into $c=3$ classes
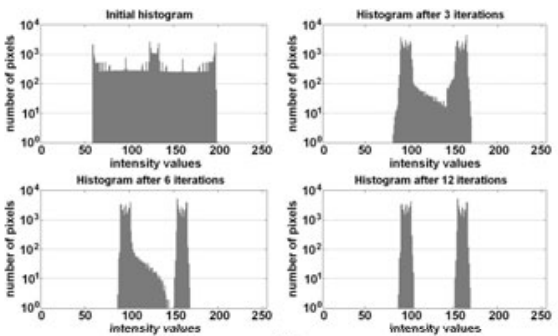

(a)

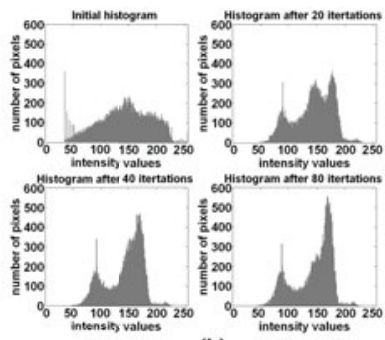

(b)

Fig. 2. (a) The evolution of the histogram of an INU contaminated two-class phantom image, during the iteration cycles; (b) The evolution of the intensity distribution of an INU contaminated real MR image during the iteration cycles

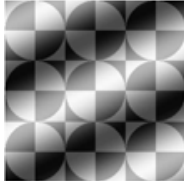

(a)

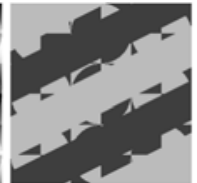

(b)

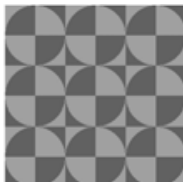

(c)

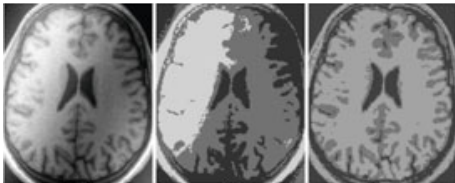

(a) (b)

Fig. 3. Segmentation of phantoms (left) and real MR images (right): (a) original image, (b) failed segmentation without INU compensation, (c) successful segmentation with INU compensation 
image. Real MR brain images of different sizes, artificially contaminated with inhomogeneity were also fed to both algorithms. These images were segmented into $c=3$ classes corresponding to white matter (WM), gray matter (GM), and cerebro-spinal fluid (CSF), respectively. Benchmark results are exhibited in Fig. 1(b). The speed-up ratio is even higher: it varies between 28 and 32 .

Figure 2(a) shows the variation of the histogram of a phantom image during the iterative compensation. The two classes are perfectly separated after 10-12 iteration cycles. The regions the compensation produces are not piecewise constant, but they are easily separable, so the classification can be $100 \%$ accurate. Figure2(b) shows the variation of the histogram of a brain MRI image segmented into three clusters, during the iterative compensation. Here convergence requires 30-80 iterations. As the WM and GM have their intensities close to each other, in the presence of noise their histograms overlap, so their distributions cannot be completely separated by INU compensation. This is a primary source of misclassifications, equally present in both the conventional and proposed approaches.

Figure 3 exhibits the segmentation and INU compensation of a two-class phantom, and a real MR image. Without compensation the segmentation fails, but compensation makes the classes perfectly separable. The beneficial effect of INU compensation is also visible in real MR images, but here tissues are not perfectly separable due to their overlapping intensity ranges.

As $c \ll \omega \ll n$, the theoretical complexity values exhibited in Table 2 suggest that the running time of the accelerated algorithm hardly depends on the number of clusters. To confirm this hypothesis, we have fed various images to the algorithm, setting the number of clusters $c$ to values ranging from 2 to 8 . The obtained speed-up ratios linearly grew together with $c$, reaching 65 at $c=8$.

Both approaches theoretically perform the same computations, so the accelerated approach should have exactly the same accuracy as the conventional one. However, in practice, there is a secondary source of errors in the proposed approach due to the quantification error of the bias field. In our tests, bias quantification never increased the number of misclassifications more than $1 \%$.

The proposed method is also compatible with other INU modeling schemes: in case of modeling INU as gain field, or in the log-bias approach, the partition and cluster prototypes can be computed using the histogram, just as we have shown in the bias field formulation. The proposed approach is insensitive to the chosen bias smoothing procedure: the user has the freedom to apply for example the mean spread filtering [10]. Further on, the formulation of the proposed solution is adaptable to other $c$-means clustering algorithms [13.

\section{Conclusion}

In this paper, we have reformulated the $c$-means clustering based approach of INU compensation and segmentation of magnetic resonance images, in order to drastically reduce the processing time. We have shown that the most time consuming parts of the conventional algorithm's iteration cycle can be applied to individual gray intensities instead of individual pixels. We achieved an approach 
that performs the segmentation of brain MR images 20-30 times faster, without causing relevant change in terms of accuracy. The proposed algorithm is highly compatible with various reported $c$-means clustering based INU compensation techniques. With this increased execution speed, $c$-means clustering may receive a significantly higher popularity in the domain of MR image segmentation.

\section{References}

1. Ahmed, M.N., Yamany, S.M., Mohamed, N., Farag, A.A., Moriarty, T.: A modified fuzzy $c$-means algorithm for bias field estimation and segmentation of MRI data. IEEE Trans. Med. Imag. 21(3), 193-199 (2002)

2. Bezdek, J.C.: Pattern recognition with fuzzy objective function algorithms. Plenum, New York (1981)

3. Derganc, J., Likar, B., Pernuš, F.: Nonparametric segmentation of multispectral MR images incorporating spatial and intensity information. Progr. Biomed. Opt. Imag. 3(1), 391-400 (2002)

4. Internet Brain Segmentation Repository, http://www.cma.mgh.harvard.edu/ibsr

5. Johnston, B., Atkins, M.S., Mackiewich, B., Anderson, M.: Segmentation of multiple sclerosis lesions in intensity corrected multispectral MRI. IEEE Trans. Med. Imag. 15(2), 154-169 (1996)

6. Liew, A.W.C., Hong, Y.: An adaptive spatial fuzzy clustering algorithm for 3-D MR image segmentation. IEEE Trans. Med. Imag. 22(9), 1063-1075 (2003)

7. Pal, N.R., Pal, K., Keller, J.M., Bezdek, J.C.: A possibilistic fuzzy c-means clustering algorithms. IEEE Trans. Fuzzy Syst. 13(4), 517-530 (2005)

8. Pham, D.L., Prince, J.L.: Adaptive fuzzy segmentation of magnetic resonance images. IEEE Trans. Med. Imag. 18(9), 737-752 (1999)

9. Rajapakse, J.C., Kruggel, J.C.: Segmentation of MR images with intensity inhomogeneities. Image Vis. Comput. 16(3), 165-180 (1998)

10. Siyal, M.Y., Yu, L.: An intelligent modified fuzzy $c$-means based algorithm for bias field estimation and segmentation of brain MRI. Patt. Recogn. Lett. 26(13), 2052-2062 (2005)

11. Sled, J.G., Zijdenbos, A.P., Evans, A.C.: A nonparamtertic method for automatic correction of intensity nonuniformities. IEEE Trans. Med. Imag. 17(1), 87-97 (1998)

12. Styner, M., Brechbuchler, C., Székely, G., Gerig, G.: Parametric estimate of intensity inhomogeneities applied to MRI. IEEE Trans. Med. Imag. 19(3), 153-165 (2000)

13. Szilágyi, L., Szilágyi, S.M., Benyó, B., Benyó, Z.: Intensity inhomogeneity compensation and segmentation of MR brain images using hybrid $c$-means clustering models. Biomed. Sign. Proc. Contr. 6(1), 3-12 (2011)

14. Vemuri, P., Kholmovski, E.G., Parker, D.L., Chapman, B.E.: Coil sensitivity estimation for optimal SNR reconstruction and intensity inhomogeneity correction in phased array MR imaging. In: Christensen, G.E., Sonka, M. (eds.) IPMI 2005. LNCS, vol. 3565, pp. 603-614. Springer, Heidelberg (2005)

15. Vovk, U., Pernus, F., Likar, B.: MRI intensity inhomogeneity correction by combining intensity and spatial information. Phys. Med. Biol. 49(17), 4119-4133 (2004)

16. Vovk, U., Pernuš, F., Likar, B.: A review of methods for correction of intensity inhomogeneity in MRI. IEEE Trans. Med. Imag. 26(3), 405-421 (2007)

17. Zhang, Y., Brady, M., Smith, S.: Segmentation of brain MR images through a hidden Markov random field model and the expectation-maximization algorithm. IEEE Trans. Med. Imag. 20(1), 45-57 (2001) 